\title{
Aplicabilidad del Service-Dominant Logic al ámbito sanitario: caracterizando el servicio para la co-creación de valor*
}

\section{Applicability of Service-Dominant Logic to the health sector: characterizing the service for the co-creation of value}

\section{Aplicabilidade do Service-Dominant Logic no âmbito sanitário: a caracterizar o serviço para a co-criação de valor}

Fecha de recepción: 19-08-15 Fecha de aceptación: 04-09-15 Disponible en línea: 01-11-15 doi: 10.11144/Javeriana.rgyps14-29.asdl

Cómo citar este artículo:

Calero Gimeno R, Gallarza Granizo MG. Aplicabilidad del Service-Dominant Logic al ámbito sanitario: caracterizando el servicio para la co-creación de valor. Rev. Gerenc. Polít. Salud. 2015; 14(29):

179-192 http://dx.doi.org/10.11144/Javeriana.rgyps14-29.asdl

Artículo de revisión, toma como base la tesis doctoral titulada El comportamiento de lealtad del paciente: un análisis aplicado al Modelo Alzira, leída por Remedios Calero en la Universidad Católica de Valencia el 23 de mayo del 2014, dirigida por la Dra. Martina. G. Gallarza, con la calificación de Sobresaliente cum Laude. Correspondencia: Remedios Calero Gimeno c/ Los Huertos, 3746500 Sagunto (Valencia). Correo electrónico: remedios.calero@mail.ucv.es

* Doctora por la Universidad Católica de Valencia, España. Subdirectora de Gestión Económica en la Gerencia de Atención Integrada de Albacete (Servicio de Salud de Castilla La Mancha), España. Correo electrónico: remedios. calero@mail.ucv.es

*** Doctora por la Universitat de València, Departamento de Comercialización e Investigación de Mercados de la Facultad de Economía de la Universitat de València, España. Correo electrónico: martina.gallarza@uv.es 


\section{Resumen}

Contexto: desde mediados de los años 2000, la literatura de marketing de servicios propone el Service-Dominant Logic como una novedosa visión estratégica, basada en la creación de un mayor valor con —en lugar de para - el consumidor. Este trabajo busca explorar su aplicabilidad al servicio sanitario. Metodología: mediante la revisión y discusión teórica de la especificidad del servicio sanitario bajo las premisas del Service-Dominant Logic, se han evidenciado: a) cuáles son sus principales elementos caracterizadores y, b) cómo se logra la co-creación de valor en el ámbito de este servicio. Resultados: son caracterizadores del servicio sanitario su carácter crítico, su naturaleza credence y su dimensión social. Con esta realidad, la co-creación de valor en el ámbito sanitario se obtiene de la participación activa de un paciente cada vez más informado, y de la comprensión de los complejos equilibrios de la relación médico-paciente.

Palabras clave: servicios de salud; marketing de servicios de salud; participación del paciente; relaciones médico-paciente; cooperación del paciente

\section{Abstract}

Context: since the mid-2000s, the services marketing literature proposes Service-Dominant Logic as an innovative strategic vision, based on the creation of greater value with - rather than for - the consumer. This paper seeks to explore their applicability to the health service. Methodology: by reviewing and discussing the theoretical of the specificity of the health service on the premises of the Service-Dominant Logic it has been demonstrated that: a) what its main characteristic elements are, b) how the co-creation of value is achieved in the scope of this service. Results: The credence nature, its critical character and its social dimension characterize the health service. With this reality, the co-creation of value in health care is obtained from the active participation of a patient becoming more informed and understanding of the complex balances of the doctor-patient relationship.

Keywords: health services; marketing of health services; patient participation; physician-patient relation; patient compliance

\section{Resumo}

Contexto: desde meados da década de 2000, a literatura de marketing de serviços propõe o Service-Dominant Logic como inovadora visão estratégica, baseada na criação de um maior valor com —em lugar de para - o consumidor. Este trabalho visa explorar sua aplicabilidade ao serviço sanitário. Metodologia: mediante revisão e discussão teórica da especificidade do serviço sanitário sob as premissas do Service-Dominant Logic, tem se evidenciado: a) quais os principais elementos caraterizadores e, b) como é possível a co-criação de valor no âmbito deste serviço. Resultados: são caracterizadores do serviço sanitário o seu caráter crítico, sua natureza credence e sua dimensão social. Com essa realidade, a co-criação de valor no âmbito sanitário é obtido da participação ativa de um paciente cada vez mais informado, e da compreensão dos complexos equilíbrios do relacionamento médico-paciente.

Palavras-chave: serviços de saúde, marketing de serviços de saúde, participação do paciente, relacionamento médico-paciente, cooperação do paciente 


\section{Introducción}

La idiosincrasia del servicio sanitario permite que este sea analizado, desde un prisma disciplinar de marketing de servicios, bajo algunos de las más novedosos enfoques académicos, como serían las premisas del llamado Service-Dominant Logic ${ }^{1}$ apuntadas en el Journal of Marketing y en el Journal of the Academy of Marketing Science por los profesores Steve Vargo y Robert Lusch (1,2),y discutidas y ampliadas con posterioridad por otros autores que también han desarrollado esta óptica de análisis para la mejor comprensión de la provisión de productos y servicios al mercado (3). De manera más precisa, nos referimos a las temáticas de la integración y co-creación de valor con el destinatario del servicio, la interactividad y la llamada customización o necesidad de realizar servicios a medida del cliente. Todas ellas son "lentes" a través de las cuales se puede entender mejor la prestación del servicio sanitario, en ámbitos como la creciente información del paciente y la complejidad del proceso de producción del servicio.

Esta aproximación del Service-Dominant Logic supone una visión estratégica novedosa, fuente de ventaja competitiva para las organizaciones, basada en la creación de un mayor valor con - en lugar de para - los consumidores (3). Este proceso de co-creación de valor entre oferta y demanda pivota en torno al servicio como centro de intercambio, integrando a todos los agentes y generando un valor único para cada cliente, combinando capacidades, recursos y conocimientos (2). Implica un nuevo enfoque para proveedores, consumidores e intermediarios, al resaltar el rol del consumidor como co-creador de valor $(4,5)$.

1 Traducido al español como "lógica del servicio", aunque la fórmula en inglés está muy extendida también en la literatura en castellano.
Dos de las características de esta nueva óptica que, a nuestro juicio, la hacen apropiada para comprender el servicio sanitario en su nuevo escenario, son el poder máximo que se otorga al cliente en la creación de valor, poder que se considera creciente en el caso del servicio sanitario (6), y su aplicabilidad en sistemas complejos de servicio (7). Estas son dos de las justificaciones para la propuesta de este trabajo.

De manera más concreta, tratamos de arrojar luz sobre la aplicabilidad del Service-Dominant Logic al servicio sanitario, mediante la revisión y discusión teórica de la especificidad del servicio sanitario, exponiendo primero sus principales elementos caracterizadores y, en segundo lugar, abordando la co-creación de valor en el ámbito de este servicio, por ser este el eje vertebrador de este novedoso enfoque.

\section{El servicio sanitario: revisión de sus elementos caracterizadores}

La especificidad del servicio sanitario dentro de la literatura de servicios permite considerar tres elementos caracterizadores, como muestra la figura 1: su carácter crítico, su naturaleza credence o "de confianza" y su dimensión social. Su análisis nos permite encontrar puntos de anclaje para la posterior discusión de la co-creación de valor como eje de la lógica dominante del servicio.

\section{El carácter crítico del servicio sanitario}

Una primera caracterización del servicio sanitario, con respecto a otros servicios, es su carácter crucial para el usuario. Sin embargo, su prestación está rodeada de complejidad e incertidumbre. La confluencia de estas realidades nos lleva a poder considerarlo como crítico, lo que dificulta su gestión. 


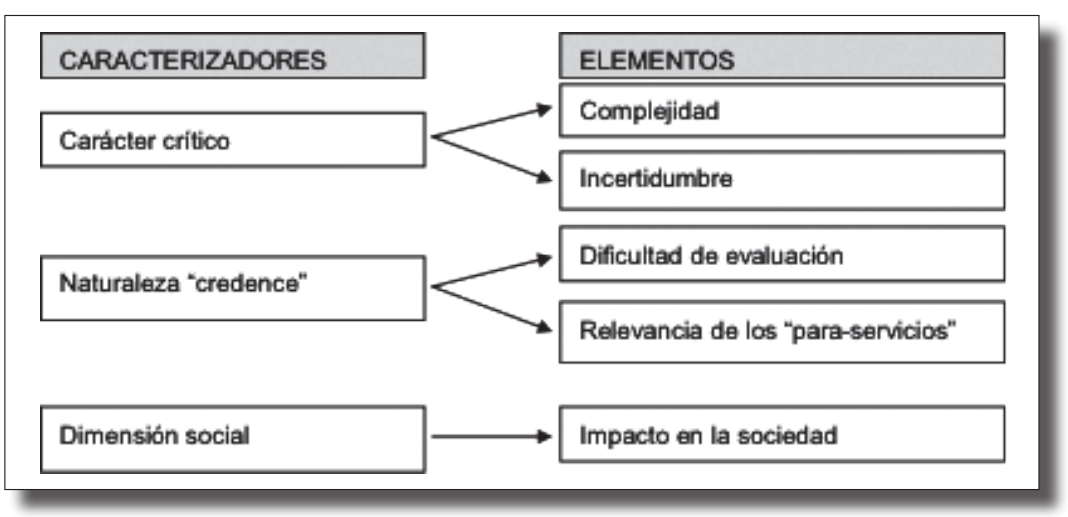

Figura 1. Caracterizadores del Servicio sanitario

Fuente: elaboración propia

La elevada complejidad en la prestación del servicio. Las organizaciones sanitarias se mueven en un escenario único en el que deben ofrecer productos y servicios a diferentes públicos - pacientes, familiares, personal sanitario- $(8,9)$. A este peculiar escenario se añade que el servicio sanitario es intensivo en personal (10) y que, como proceso, está formado por procedimientos complejos que requieren un conocimiento especializado y una elevada dotación tecnológica. Mediante estos procedimientos, se pretende resolver gran variedad de problemas concretos (alterar el curso normal de las enfermedades, prevenir, curar o rehabilitar), no exentos de dificultad en su prestación (11).

Otra fuente de complejidad reside en el hecho de que algunas partes del proceso requieran la interacción del profesional sanitario con el paciente, otras son realizadas por los propios pacientes (como tomarse la medicación), mientras que algunas fases involucran a terceras partes (ej. un laboratorio) (12).

Por otra parte, cabe considerar que el nivel de complejidad no es el mismo para todos los componentes del servicio sanitario, sino que cada servicio debe ser analizado en función de dos variables: su divergencia y su complejidad (13). La figura 2 permite ilustrar esta bidimensionalidad en un doble eje: así, en un laboratorio de radiología estas variables alcanzan valores mínimos, comparadas con el servicio prestado por un médico general, en el que debe realizar el diagnóstico y tratamiento, con altas tasas de divergencia y complejidad.

Por todo ello, el servicio sanitario debe desarrollarse en un entorno diseñado con tal detalle que permita tanto la especial interacción entre productor y consumidor como su elevada complejidad (14-17), teniendo en cuenta unas expectativas de los usuarios cercanas a la excelencia (18).

La incertidumbre que envuelve al servicio sanitario. La heterogeneidad que caracteriza a los servicios se ve aumentada cuando el producto del servicio sanitario es el paciente (19-23): indudablemente, la adaptación del servicio a las características de cada persona dificulta su estandarización. Es por ello que, en servicios caracterizados por una gran variabilidad en la demanda, como es el caso del servicio sanitario, la heterogeneidad alcanza cotas máximas. Esta elevada diversidad de la demanda a la que se enfrenta, junto con la alta disponibilidad del paciente a participar en su co-producción, incrementan el nivel de incertidumbre al que se ve expuesto el ser- 


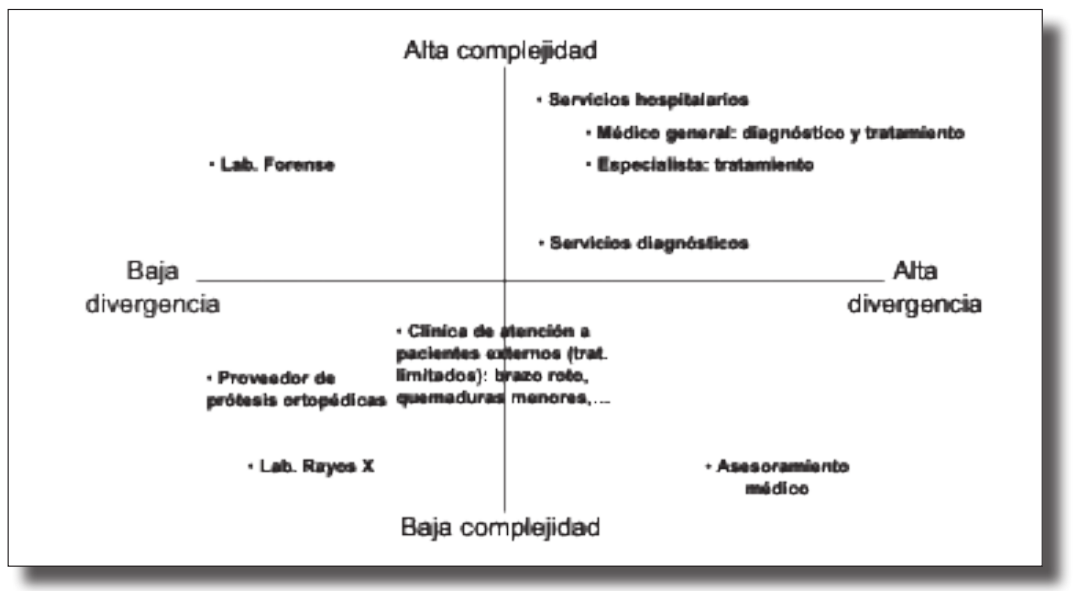

Figura 2. Complejidad y divergencia en el SERVicio SANitario

Fuente: adaptación de Lovelock (13)

vicio sanitario $(14,21)$. La incertidumbre se da tanto en el proceso como en el resultado, siendo uno de los factores que más influyen en el comportamiento de los profesionales médicos. Si consideramos el gran peso del capital humano en la provisión de servicios sanitarios, observamos cómo una parte esencial de su trabajo consiste en diagnosticar a los pacientes, a menudo con urgencia y disponiendo de información imperfecta, ya que el estado de salud de un paciente es difícil de determinar con exactitud incluso para un médico. Es por ello que, de manera habitual, el médico se enfrenta a situaciones en las que se requiere tomar decisiones con rapidez en condiciones de gran incertidumbre (13).

Todo ello lleva a Torrey (24) a hablar de la Teoría FUD (fear, uncertainty and doubt). Según esta teoría, los pacientes, como consumidores, eligen proveedores cada día. Sin embargo, la elección de centro hospitalario o profesional médico presenta una gran dificultad debido a la vulnerabilidad que siente el paciente. Esta vulnerabilidad es fruto de la incertidumbre sobre los resultados, la falta de información especializada o la situación de ansiedad presente en ciertas ocasiones. El paciente se encuentra enfermo y bajo cierto nivel de estrés y miedo (25), por lo que necesita que le aseguren que todo está bajo control. A efectos de ofrecer cierta seguridad, reduciendo esta incertidumbre y mejorando la calidad asistencial, como veremos más adelante, la gestión sanitaria trata de desarrollar estrategias de adecuación y protocolización de los diferentes procesos (26).

Esta vulnerabilidad se encuentra relacionada también con el hecho de que el servicio sanitario invade, en cierta medida, la esfera privada del paciente (25). Este se enfrenta a la tesitura de tener que tratar temas personales con el médico que probablemente pocas personas de su entorno conozcan, o que pueden no resultarle agradables de tratar (como el sobrepeso o sus hábitos nocivos). En consecuencia, los aspectos emocionales adquieren una mayor relevancia que los cognitivos en el comportamiento del consumidor en el servicio sanitario (27). Si bien durante el encuentro de servicio surgen sentimientos en el consumidor, en un servicio como el sanitario con una fuerte interacción personal, estos sentimientos se vuelven todavía más intensos $\mathrm{y}$, en ciertas ocasiones, incluso negativos (28). 
En definitiva, nos encontramos ante un servicio que debe atender a cuatro dimensiones a efectos de su prestación: psicológica (depresión, nerviosismo, preocupación ante el presente o el futuro que puede experimentar el paciente); existencial (creencias sobre la vida, la enfermedad, etc.); emocional (sentimientos del paciente y necesidad de apoyo); y física (síntomas físicos como pérdida de peso, entre otros). Estas cuatro dimensiones son de máxima relevancia en el caso de patologías como el cáncer y en tratamientos crónicos en los cuales la dimensión física cede un importante protagonismo a las otras tres dimensiones (29). Por todo ello, considerando la complejidad en su prestación y el elevado grado de incertidumbre y elementos emocionales, observamos cómo el servicio sanitario adquiere un cariz crítico.

\section{La naturaleza credence del servicio sanitario}

La dificultad de evaluación por parte del paciente. Partiendo de las categorías de atributos definidas por Nelson (30) — search qualities (cualidades de búsqueda) y experience qualities (de experiencia)—, Darby y Karni (31) añaden una tercera, credence qualities (de confianza), que recoge características imposibles de evaluar por el consumidor incluso después de la compra y el consumo del servicio, señalando la falta de conocimientos específicos como la principal causa de esta dificultad de evaluación, lo que sin duda es el caso del servicio sanitario. En esta línea, Zeithaml et al. (12) realizan una clasificación de productos y servicios en función de la facilidad o dificultad del consumidor a la hora de evaluarlos, ocupando el servicio sanitario un lugar extremo: servicios que poseen un mayor grado de características de credibilidad y confianza puesto que, incluso después de su consumo, el usuario es incapaz de evaluar el servicio: muy pocos pacientes disponen de los suficientes conocimientos médicos como 184 para valorar si el servicio sanitario que han recibido ha sido el adecuado y se ha prestado correctamente (12,32-34). Un paciente puede saber si los síntomas han desaparecido, pero no es capaz de juzgar él mismo si ha sido curado de su enfermedad o si el tratamiento prescrito constituía la alternativa más eficaz. Es por ello que la asistencia sanitaria se plantea como una obligación de medios, no de resultados, puesto que el no alcanzar el resultado deseado no conlleva que el servicio haya sido mal prestado (35). De este modo, un paciente puede sentirse insatisfecho con la atención recibida, pese a ser tratado por un equipo médico con un nivel excelente (36), debido a que no dispone de la capacidad de juzgar en qué medida fue adecuado.

\section{La especial relevancia de los "para-servicios".}

La dificultad de evaluación del servicio sanitario provoca que, más allá de la calidad clínica, los usuarios otorguen una especial valoración a otros atributos que rodean la prestación principal. Los llamados "para-servicios": el trato personal, la empatía, la seguridad, la consistencia, la actitud del profesional, el entorno, la disponibilidad, la competencia, la profesionalidad y la rapidez $(22,37,38)$. Así, la demanda de los ciudadanos no se basa únicamente en la obtención de un servicio sanitario eficaz, sino también en un trato personal y humano agradables $(37,38)$.

A todos estos atributos, hay que añadir la elevada valoración que otorgan los pacientes a elementos tangibles tales como el equipamiento o el alojamiento (12). De esta manera, Lovelock (13, p. 56) se pregunta “iqué impresión causa en un futuro usuario que visita a un familiar en un hospital el encontrar un edificio pulcro y bien conservado, bien decorado y con un plantilla atenta y amable?”. Esta cuestión reafirma la idea de que, al no disponer de conocimientos suficientes, el paciente tiende a valorar el servicio sanitario a través de factores que puede tangibilizar. Cobra (39, p. 18) ilustra esta idea mediante la siguiente metáfora: "Los aspectos tangibles de un servicio son como la parte visible de un iceberg: lo que 
está por encima del nivel del mar equivale al nivel consciente de las personas; los aspectos intangibles son como la parte sumergida del iceberg, invisibles a los ojos del consumidor y en el subconsciente e inconsciente de las personas". De este modo, se pone en evidencia el esfuerzo que debe realizar el gestor sanitario en comunicación e información al paciente, a efectos de no chocar con ese iceberg.

\section{La dimensión social del servicio sanitario}

El servicio sanitario tiene un componente sensiblemente social provocado por su relación inseparable con las necesidades de las personas $(13,14,39)$, de tal manera que el objetivo del servicio sanitario debe ser la provisión de la mejor salud posible al máximo de población (34). Es decir, una mayor cantidad de servicios ofrecidos no equivale necesariamente a la provisión de un mejor servicio sanitario. De esta manera, si el objetivo del servicio sanitario es la provisión de la mejor salud posible, la prestación de este servicio tendrá una repercusión directa en el nivel de salud de la población.
En esta dimensión social, podemos distinguir entre pacientes y ciudadanos, siendo estos últimos usuarios potenciales de los servicios sanitarios. A diferencia de lo que ocurre con otros servicios, la relevancia y opinión de los ciudadanos no reside en su uso del servicio sanitario, sino en la preocupación que manifiestan por el sistema y el servicio sanitario deseado para la sociedad en su conjunto. Por todo ello, podemos considerar que el servicio sanitario trasciende el acto de provisión de un servicio en particular para convertirse en un concepto social.

\section{La co-creación de valor en el ámbito sanitario}

A continuación, mostramos la relación existente entre los caracterizadores del servicio sanitario descritos y lo que en la óptica del Service-Dominant Logic se entiende como elementos facilitadores de la co-creación de valor, que en el ámbito sanitario serían la participación del paciente y la relación médico-paciente (cfr. figura 3).

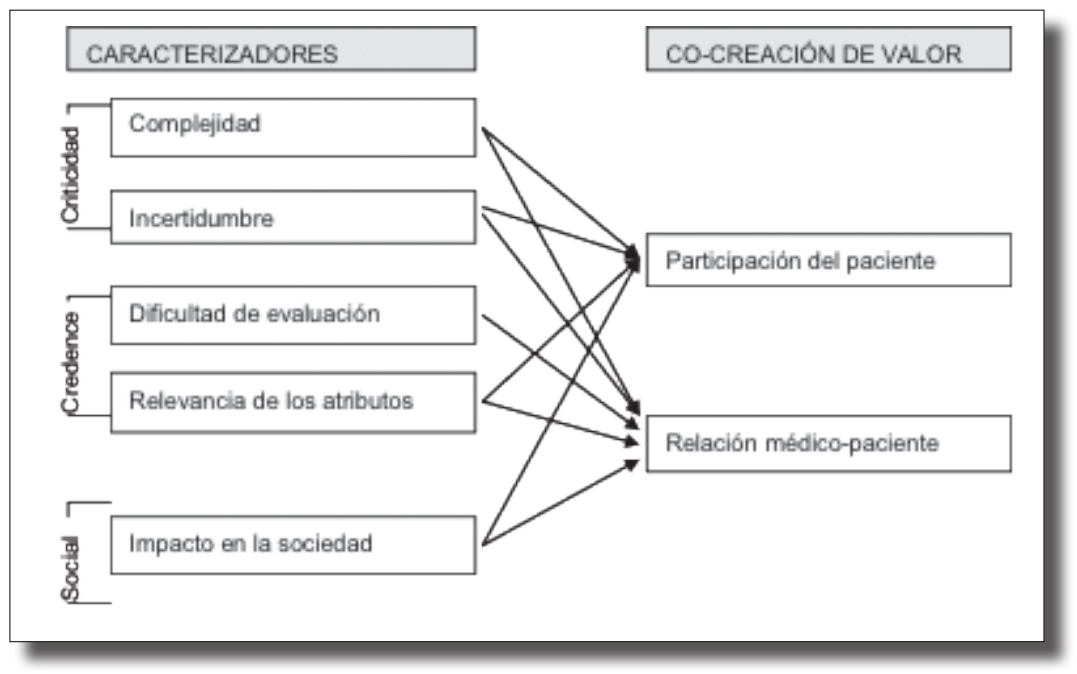

Figura 3. RELACIÓN ENTRE LOS CARACTERIZADORES DEL SERVICIO SANITARIO Y LA CO-CREACIÓN DE VALOR 


\section{La participación del paciente}

El caso extremo de simultaneidad entre producción y consumo lo constituyen los servicios personales, en los que el cliente debe estar presente durante todo el proceso de producción, como por ejemplo un paciente durante una cirugía (22). En esta visión, la participación del paciente en la producción del servicio sanitario constituye un elemento crítico en la experiencia de consumo (40). El hecho de que el proceso de producción del servicio tenga lugar en presencia del paciente implica que las distintas etapas de producción tienen su efecto directo sobre este al formar parte de la programación, diseño y co-creación del servicio (14). Mediante esta participación, los usuarios se convierten en co-creadores y co-productores del servicio, más allá del simple rol de consumidores $(14,32,41)$. Adicionalmente, la participación del paciente en la toma de decisiones aumenta su percepción de calidad del servicio, especialmente en aquellos servicios con un elevado riesgo percibido $(42,43)$ e incertidumbre (26) es el caso del sanitario.

En este sentido, la participación del paciente puede entenderse también desde un prisma de corresponsabilidad (44). Los distintos cambios sociales, culturales, políticos y éticos están llevando a las profesiones sanitarias a evolucionar hacia un modelo centrado en el paciente, en el que tiene lugar una decisión compartida. Así, se acuña el término medicina deliberativa para diferenciar de las soluciones técnicas, que no requieren la implicación del paciente en la solución definitiva, aquellas soluciones adaptativas que, al ser más complejas, sí requieren de la aportación de este (45).

Esta participación máxima del cliente es determinante en la visión del Service-Dominant

186 Logic. Aplicado al servicio sanitario, Karpen et al. (3) citan como ejemplo el caso de una consulta médica. En ella, el doctor toma nota de las condiciones médicas del paciente y su entorno, ofrece apoyo emocional y psicológico, transmite al paciente los riesgos del tratamiento e informa y comparte detalles al respecto. Al mismo tiempo, el médico requiere un feedback del paciente, como una co-participación en la creación de valor. De manera adicional, podrían citarse otras actividades co-creadoras de valor, tanto preventivas como curativas, con una elevada participación del paciente tales como la adopción de estilos de vida saludables, la realización de pruebas de detección precoz de enfermedades o el fiel seguimiento de los pasos indicados para reducir la complejidad y severidad de los tratamientos (42).

En consecuencia, la co-creación de valor puede darse en todos los niveles (46), siendo posible identificar, entre otras, una serie de actividades en las que tiene lugar esta co-creación (29): cooperación; contraste de información; combinación de terapias complementarias; co-aprendizaje; cambio en las formas de hacer las cosas; conexión con familiares, amigos, doctores y otros profesionales; co-producción en el diseño de tratamientos; $y$ actividades cerebrales como el pensamiento positivo. Todas estas actividades se caracterizan por una fuerte implicación y participación del paciente que conlleva una co-creación de valor en la prestación.

Del mismo modo que pueden identificarse actividades en las que tiene lugar una cocreación de valor, es posible diferenciar estilos de pacientes en su intervención como co-creadores de valor $(29,47)$. La distinción realizada por este último autor se basa en el nivel de participación del paciente en actividades y en su interrelación con otros actores (cfr. figura 4.): gestión de equipos, control aislado, acompañamiento, adaptación pragmática y conformidad pasiva. Así, en la gestión de equipos, el paciente se implica 
de tal modo que interviene en un elevado número de actividades con gran cantidad de interacciones con otros actores del proceso. Por el contrario, en la conformidad pasiva el paciente apenas interviene en las actividades y no se interrelaciona con el resto de actores, únicamente sigue las órdenes del doctor. El estilo de acompañamiento se caracteriza por un nivel medio de actividades e interacciones del paciente. Por su parte, en la adaptación pragmática, el paciente participa en pocas actividades mientras que su interacción con otros actores del proceso es muy elevada. En el sentido opuesto, en el control aislado el paciente se involucra en gran número de actividades, pero se muestra incapaz de interrelacionarse.

Por todo ello, puede afirmarse que los pacientes no muestran siempre el mismo grado de co-creación de valor del servicio sanitario, sino que pueden desempeñar diferentes roles. Esta disparidad de roles es un aspecto que es necesario considerar en la gestión del servicio sanitario, a fin de ofrecer el mejor servicio al paciente.

\section{La relación médico-paciente}

La especial relación médico-paciente es resultado de la propia idiosincrasia del servicio sanitario: un servicio personal, en el que tiene lugar una relación de agencia entre los actores y en el que el médico como prestador del servicio desempeña un rol esencial.

Relación de agencia. Una primera cualidad que da forma a la relación médico-paciente es que esta relación no es de igual a igual, lo que la literatura denomina como relación de “agencia” (23,48). El paciente acude en busca del conocimiento y pericia del profesional, a lo que se suma que en la enfermedad hay una posición de partida del paciente incómoda, teñida muchas veces por la angustia y el temor (relacionado con la criticidad e incertidumbre, características anteriormente señaladas). En el sector sanitario, la peculiaridad de la relación hace que el personal sanitario adquiera una mayor relevancia e intensidad que en cualquier otro servicio. De este modo, la relación médico-paciente está dotada de un componente psicológico, con una gran confianza del paciente en el

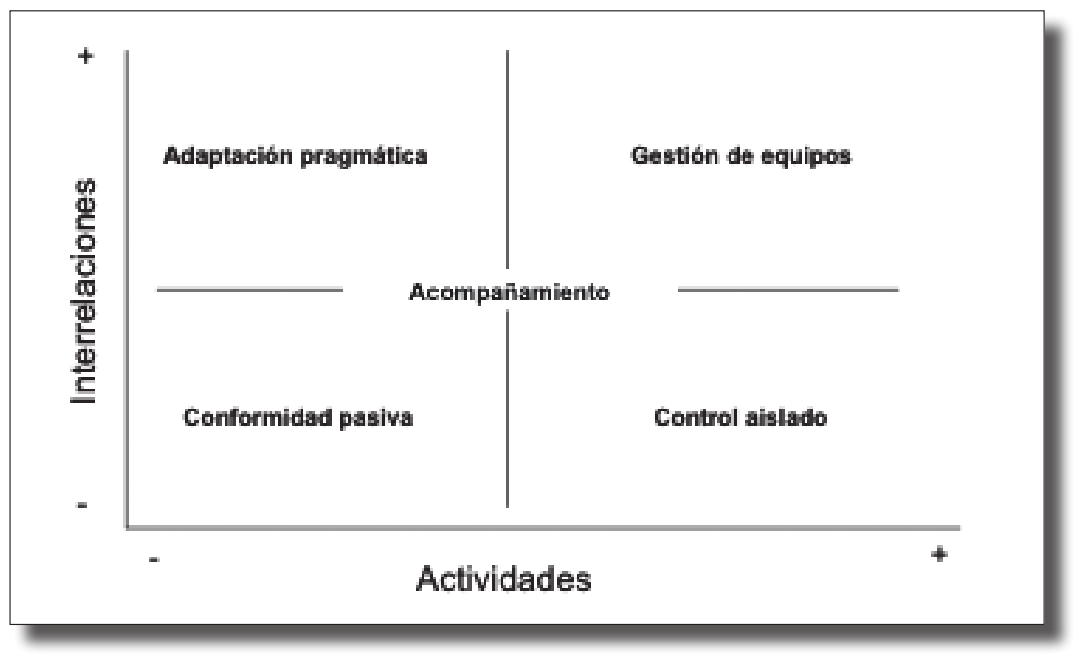

Figura 4. Estilos de Co-Creación de valor del paciente 
criterio del doctor (32,33). Nos encontramos, así, ante una situación de dependencia (13). Esta desigualdad tiene como origen, en parte, la falta de conocimientos específicos del paciente que le aboca a depender del consejo del profesional sanitario a la hora de tomar decisiones $(49,50)$, especialmente en situaciones delicadas o revestidas de cierta gravedad. Estos miedos pueden llevar al paciente a mostrarse incapaz de preguntar al doctor por detalles del tratamiento o por los resultados que cabe esperar. Es más, incluso puede llegar a considerarse atrevido o poco correcto el preguntar en demasía al profesional sanitario (42).

Así, distintos autores que consideran la relación entre médico y paciente como una relación de "agencia", siendo los flujos de información esenciales en esta relación $(23,48)$. Precisamente, Errasti (20, p. 202) define la relación médico-paciente como el intercambio en el que "el paciente comunica los datos pertinentes y recibe información acerca de la naturaleza de su enfermedad y el tratamiento". El médico posee una mejor posición respecto al paciente en el dominio de la información sobre la indicación y la efectividad de los procedimientos disponibles $(32,42,51)$. A priori, el mercado resulta incompetente a la hora de proveer información completa, debido a que la información requerida en este servicio se caracteriza por ser individual e intransferible, con un elevado coste de adquisición, una naturaleza técnica que no facilita la comprensión del usuario y unos costes de una decisión equivocada muy elevados. De este modo, al carecer de información suficiente, la soberanía del consumidor se ve limitada (51).

\section{Cambios en la gestión de la información en} el marco de la relación médico-paciente. No obstante, pese a esta relación de agencia, la posibilidad de acceso a gran cantidad de información médica está cambiando la relación médico-paciente, con un paciente más informado sobre su enfermedad. Por un lado, la información ofrecida por los distintos medios de comunicación influye tanto en la conducta del paciente como en la toma de decisiones de este (27). Por otro, el paciente muestra una actitud proactiva, recurriendo a fuentes de información como Internet (52-54). Surge, en este último caso, el auge del llamado $e$-healthcare $(55,56)$, del que se observa un notable interés en la literatura por el estudio de sus efectos sobre el ya complejo encuentro de servicio sanitario. Así, Zeithaml et al. (12, p. 405) califican como health seekers ${ }^{2}$ a aquellos pacientes que se preocupan por buscar información en la red sobre enfermedades específicas, nutrición, fármacos, etc. Precisamente, esta búsqueda online les facilita información rápida de forma anónima.

En este sentido, una de las principales conclusiones de esta evolución del paciente reside en que el profesional sanitario pierde cierta legitimidad ante un paciente con un acceso sin precedentes a información técnica especializada, permitiéndole cuestionar el servicio recibido (57). Se establece una nueva relación de poder. No estamos ante un usuario pasivo, sino ante un paciente más informado, preocupado por su salud y dotado de un mayor nivel de autonomía $(21,58)$. Se trata, pues, de un paciente que no se conforma con recibir toda la asistencia sanitaria en un mismo centro, sino que se muestra capaz de discriminar por tipo de asistencia y acudir a aquel centro que considera más adecuado en función de la patología que es preciso tra$\operatorname{tar}(42)$, lo que afecta directamente de cara a la implementación de modelos de gestión de base capitativa y financiación intercentros, en los que se otorga libertad de elección de centro y médico al paciente.

2 Traducido al español como "buscadores de información". 
Esta nueva realidad se encuentra en consonancia con los principios teóricos de la cocreación de valor (1), que facilita una mayor orientación al cliente en el diseño y producción del servicio, pero también necesita ser asimilada y aprehendida por el prestador del servicio, llevando al desempeño de un nuevo rol del profesional sanitario, en el que debe estar más preparado para responder a un paciente más informado (59). Precisamente, en esta línea, encontramos en la literatura una corriente de autores que apuestan por ofrecer información al paciente en todos los niveles de atención $(34,42,60)$.

\section{Rol desempeñado por el profesional sanita-} rio. El profesional sanitario ocupa un lugar central en la co-creación de valor, puesto que tiene que lidiar con consumidores más informados y sofisticados, pero recae en él la facultad de decisión sobre distintos factores como el tiempo de dedicación a cada paciente, qué pruebas diagnósticas se realizarán, qué tratamiento se prestará al paciente, qué recursos empleará, etc. Su papel va más allá de la asistencia sanitaria, resultando un agente fundamental del proceso, con capacidad de gestionar recursos, velar por el conocimiento, modular la demanda y garantizar la calidad del servicio $(32,45,59)$. Se convierte, así, en intermediario y prescriptor respecto de otros servicios sanitarios $(9,11,36,61,62)$, jugando pues un rol predominante. Este rol del profesional sanitario como prescriptor es resaltado por Kotler y Clarke (61, p. 16) al citar que "los gestores sanitarios argumentaban que no son los pacientes quienes eligen el hospital, sino que son los médicos quienes lo eligen. En consecuencia, el marketing, para ser efectivo, debería dirigirse a los médicos”. De tal modo que el médico utiliza su información acerca de las características y preferencias del paciente y su juicio acerca de sus carencias sanitarias y la tecnología disponible, y decide su demanda. En este contexto, a la hora de decidir su demanda, la priorización debe conciliar los intereses del paciente, los de los demás pacientes bajo la responsabilidad del mismo médico y los suyos propios, asociados con su retribución y otras dimensiones de satisfacción y estímulo (51).

Asimismo, se da una relación continuada entre médico y paciente, lo que exige cierto nivel de adaptación entre ellos (63), de tal manera que esta fuerte interacción así como la experiencia vivida por el paciente resultan elementos clave de su satisfacción (64). En esta visión, se resalta la relevancia de una buena comunicación entre ambos agentes que permita que el paciente se sienta parte del proceso de la toma de decisiones favoreciendo, de este modo, la co-producción mencionada (65). Así pues, este autor apuesta por la empatía, la compasión, el entendimiento y la cercanía como herramientas del profesional sanitario de cara a su trato con el paciente. Precisamente, el paciente no cuestiona la profesionalidad del médico o enfermero, sino que busca en esta relación otros valores no menos importantes como son la confianza, el trato personal, la empatía y la información (58). En este sentido, este autor destaca la relevancia que tiene para el paciente el sentirse cómodo contando sus problemas, preguntando sus dudas, el sentirse escuchado y tratado de forma personalizada, la rapidez en la solución a sus problemas y la posibilidad de recibir información completa y comprensible sobre su diagnóstico y/o tratamiento. Se trata, pues, de valores que van más allá del conocimiento científico para dotar a esta relación de criterios sociales y de comportamiento que definen una auténtica experiencia de servicio (28).

\section{Conclusiones derivadas de la discusión teórica}

La percepción del paciente como consumidor del servicio sanitario está evolucionando des- 
de un rol pasivo a una visión de co-creador de valor, resultando un campo paradigmático para la aplicación del Service-Dominant Logic (1). Esta propuesta constituye una de las ópticas de la gestión de los servicios más novedosas y con mayor impacto en la investigación de marketing, cuya aplicación todavía es incipiente en el ámbito sanitario.

Por todo ello, esta óptica permite una aproximación más sistemática y analítica a los complejos procesos de la prestación sanitaria, donde los equilibrios de información y competencia se han visto alterados. Al considerar a todos los agentes como generadores de valor, se sitúa al servicio en el centro de la prestación, creando valores altamente individualizados. En consecuencia, "entender cómo se produce esta co-creación en el marco del servicio sanitario es fundamental no sólo para el individuo sino también para otros actores como los profesionales médicos, las organizaciones sanitarias o las autoridades gubernamentales" (29, p. 7). Tomando en consideración los caracterizadores descritos en este trabajo y su relación con los elementos facilitadores de la co-creación de valor, se evidencia la utilidad y se justifica la aplicabilidad del Service-Dominant Logic al servicio sanitario. Del mismo modo, se ponen de manifiesto sus implicaciones de cara a la implementación de modelos de gestión sanitaria y atención a los pacientes: consideración del paciente en el diseño y prestación de la oferta sanitaria, con un especial cuidado e inversión en la información facilitada, ya que nos encontramos con un usuario más capacitado para seleccionar el profesional y el centro sanitario donde desea ser atendido. Todo ello repercute directamente en la implementación de modelos de gestión sanitaria, especialmente en aquellos de base capitativa y financiación intercentros, en los que se otorga libertad de elección de centro y médico al paciente, e incluso en la evaluación y aceptación del paciente de modelos de colaboración público-privada. De este modo, conocer la especial idiosincrasia del servicio sanitario y el modo en que el paciente es cocreador de valor resulta de especial interés tanto para investigadores como para gestores del servicio sanitario.

Por último, cabe señalar que este trabajo posee la limitación de ser solo una discusión teórica, invitando a futuras discusiones más aplicadas (que busquen la idoneidad de estos planteamientos quizá por especialidades médicas), y abriendo la perspectiva hacia propuestas empíricas de medición de esta co-creación de valor.

\section{Referencias bibliográficas}

1. Vargo S, Lusch RF. Evolving to a new dominant logic for marketing. Journal of Marketing. 2004; 68 (1): 1-17.

2. Vargo SL, Lusch RF. Service-Dominant Logic: continuing the evolution. Journal of the Academy of Marketing Science. 2008; 36 (1): 1-10.

3. Karpen IO, Bove LL, Lukas BA. Linking ServiceDominant Logic and strategic business practice: a conceptual model of a service-dominant orientation. Journal of Service Research. 2012; 15 (1): 21-38.

4. Ballantyne D, Varey RJ. The Service-Dominant Logic and the future of marketing. Journal of the Academy of Marketing Science. 2008; 36: 11-14.

5. Sheth JN, Sharma A The impact of the product to service shift in industrial markets and the evolution of the sales organization. Industrial Marketing Management. 2008; 37: 260-9.

6. Stanley S. 20:20 Vision: A view from the Chair. International Journal of Medical Marketing. 2002; 57.

7. Alter S. Service Systems and Service-Dominant Logic: partners or distant cousins? Journal of Relationship Marketing. 2010; 9: 98-115.

8. MacStravic RS. Product-line administration hospitals. Health Care Marketing Review. 1986; 11: 35-43.

9. Hafer JC. Developing and operationalizing a product/service classification system for health care providers. Journal of Health Care Marketing. 1987; 7 (3): 25-36.

10. Uhl KP, Upah GD. The marketing of services: why and how is it different? Illinois: University of Illinois, Working Paper 584; 1979.

11. Lamata F, Conde J, Martínez B, Horno M. Marketing sanitario. Ediciones Díaz de Santos; 1994.

12. Zeithaml V, Bitner MJ , Gremler D. Services marketing. Integrating customer focus across the 
firm, sexta edición. Nueva York: McGraw-Hill International Edition; 2013.

13. Lovelock CH. Services Marketing. Prentice Hall International Editions; 1996.

14. Bateson J. Management Services Marketing. The Dryden Press. Harcourt Brace College Publishers; 1995.

15. Casas M. Los sistemas de clasificación de pacientes. En: Jiménez J, editor. Manual de gestión para jefes de servicios clínicos. Madrid: Díaz de Santos; 2000. pp. 429-42.

16. Errasti F. Marketing y comunicación en el hospital. En Jiménez J, editor. Manual de gestión para jefes de servicios clínicos. Madrid: Díaz de Santos; 2000. pp. 85-116.

17. Medina P. El carácter estratégico de la proyección externa de la marca hospitalaria: el caso de Mayo Clinic. Universidad y Salud. 2011; 13 (1): 79-86.

18. Corella JM. Introducción a la gestión de marketing en los servicios de salud. Pamplona, España: Gobierno de Navarra, Departamento de Salud; 1998.

19. Lovelock $\mathrm{CH}$. Classifying services to gain strategic marketing insights. Journal of Marketing. 1983; 47: 9-20.

20. Errasti F. Principios de gestión sanitaria. Madrid: Díaz de Santos; 1997.

21. Oliver RL. Satisfaction: a behavioural perspective on the consumer. Singapore: McGraw-Hill; 1997.

22. Palmer A. Principles of Services Marketing. McGraw-Hill; 2005.

23. Ortún V. Las claves de la sanidad futura: investigación y gestión. Panorama Social, no 7. Ed. Funcas; 2008.

24. Torrey T. Patients, fears and engagement. En: Boyer C, Dunlop D, Stevens D, Stremcha J, Teach $\mathrm{D}$, et al., editores. The thought leaders project: hospital marketing. Minneapolis: Bierbaum Publishing: 2011. pp. 166-171.

25. Berry LL, Bendapudi N. Health Care, a fertile field for service research. Journal of Service Research. 2007; 10 (2): 111-22.

26. Peiró S. Desde las variaciones en la práctica médica a la toma de decisiones compartida y la atención centrada en el paciente. Semergen. 2003; 29 (6): 285-9.

27. Rosenstock IM. Why people use health services? The Milbank Quarterly. 2005; 83 (4): 1-32.

28. Schmitt BH. Experiential marketing. Nueva York: The Free Press: 1999.

29. McColl-Kennedy JR, Vargo SL, Dagger TS, Sweeny JC, Van Kasteren Y. Health care customer value cocreation practice styles. Journal of Service Research. 2012 May 1; 1-20.

30. Nelson P. Information and consumer behavior. The Journal of Political Economy. 1970; 78: 311-29.

31. Darby MR, Karni E. Free competition and the optimal amount of fraud. Journal of Law and Economics. 1973; 16 (1): 67-88.

32. Vertinsky IB, Thompson WA, Uyeno D. Measuring consumer desire for participation in clinical decision making. Health Services Research. 1974; 9 (2): 121-134.
33. Gabbott M, Hogg G. Consumers and services. John Wiley \& Sons; 1998.

34. Kaplan RM, Babad YM. Balancing influence between actors in healthcare decision making. BMC Health Services Research. 2011; 11: 1-14.

35. Villar Rojas FJ. La Ley de Habilitación de Nuevas Formas de Gestión del Sistema Nacional de Salud: ¿hacia la desregulación de la Sanidad Pública? Derecho y Salud. 1998; 6 (2): 74-91.

36. Gilligan C, Lowe R. Marketing and health care organizations. Nueva York: Radcliffe Medical Press; 1995.

37. Mira JJ, Rodríguez-Marín J, Peset R, Ybarra J Pérez-Jover V, Palazón I, Llorca E. Causas de satisfacción y de insatisfacción de los pacientes en hospitales y atención primaria. Revista de Calidad Asistencial. 2002; 17 (5): 273-83.

38. Molina-López M. Caja Castilla La Mancha: vinculando tecnologías e innovación sanitarias. En: Las claves de la sanidad futura: investigación y gestión; Ed. Funcas; 2008. pp. 116-20.

39. Cobra M. Marketing de servicios. Madrid: McGraw-Hill; 2000.

40. Podestà S, Addis M. Converging industries through experience. En: Carù $\mathrm{A}$, Cova $\mathrm{B}$, editores. Consuming experience. Nueva York: Routledge; 2007. pp. 139-53.

41. Barnes M. Users as citizens: collective action and the local governance of welfare. Social Policy \& Administration. 1999; 33 (1): 73-90.

42. Porter ME, Teisberg E. Redefining health care. Boston: Harvard Business Review Press; 2006.

43. Heskett JL, Sasser WE, Schlesinger LA. The service profit chain. Nueva York: The Free Press; 1997.

44. Murray E, Pollock L, White M, Lo B. Clinical decision-making: patients' preferences and experiences. Patient Education Counselling. 2007; 65: 189-96.

45. Colomer J. La gestión sanitaria a través de la administración pública: burocracia y privilegios. Gestión Clínica y Sanitaria. 2009; 4: 140-4.

46. Gaminde I. La participación de los pacientes. Revista de Calidad Asistencial. 2008; 23 (3): 93-94.

47. Aibar C. ¿Nos creemos de verdad la necesidad de participación del paciente? Revista de Calidad Asistencial. 2009; 24 (5): 183-4.

48. Gummerson E. Total Relationship Marketing. Oxford: Butterworth-Heinemann; 2008.

49. Calnan M. Citizens' views on health care. Journal of Management in Medicine. 1995; 9 (4): 17-23.

50. Gervás J. Gobierno clínico de la clínica diaria. En Ortún V, editor. El buen gobierno sanitario. Madrid: Springer Healthcare Communications; 2009. pp. 27-46.

51. Artells JJ. Necesidad vs. demanda sanitaria. Barcelona: Fundación Salud, Innovación y Sociedad; 1999.

52. Tanenbaum SJ. Consumer Perspectives on Information and Other Inputs to Decision-Making: Implications for Evidence-Based Practice. Community Mental Health Journal. 2008; 44: 331-5.

53. Sawyer SH. Market segmentation, digital campaigns and implications for the strategic marketer. 
In Boyer C, Dunlop D, Stevens D, Stremcha J, Teach D, et al., editores. The thought leaders project: hospital marketing. Minneapolis: Bierbaum Publishing; 2011. pp. 47-56.

54. Ziady N. Social media and the succession of search social media optimization. En Boyer C, Dunlop D, Stevens D, Stremcha J, Teach D. et al., editor. The thought leaders project: hospital marketing. Minneapolis: Bierbaum Publishing; 2011. pp. 57-8.

55. Lin B, Umoh D. E-healthcare: a vehicle of change. American Business Review. 2002; 20 (2): 27-32.

56. Mukherjee A, McGinnis J. E-healthcare: an analysis of key themes in research. International Journal of Pharmaceutical and Healthcare Marketing. 2007; 1 (4): 349-63.

57. Costa G. Posicionamiento de los servicios de salud en la mente del consumidor. Revista Portuguesa de Marketing. 2009; 21: 33-42.

58. Civera M. Análisis de la relación entre calidad y satisfacción en el ámbito hospitalario en función del modelo de gestión establecido [tesis doctoral]. Universitat Jaume I; 2008.
59. Pérez Somalo F. Marketing en tecnología sanitaria. Madrid: EsIC Ediciones; 2008.

60. McGlynn EA, Asch SM, Adams J, Keesy J, Hicks J, DeCristofaro A, et al. The quality of health care delivered to adults in the United Status. The New England Journal of Medicine. 2003; 348: 2635-45.

61. Kotler P, Clarke RN. Marketing for health care organizations. New Jersey: Prentice-Hall; 1987.

62. Junquera A, García Núñez RM. Marketing de productos farmacéuticos. En Cubillo JM, Cerviño J, editores. Marketing sectorial. Madrid: EsIC Editorial; 2008. pp. 285-324.

63. Barlow J, Maul D. Valor emocional en el servicio. México: Compañía Editorial Continental; 2003.

64. Henthorne TL, Salgaonkar P, George BP. External recommendations versus internal satisfaction in health care: a case study in India. Health Marketing Quarterly. 2009; 26 (3): 241-50.

65. Hollis RS. Caring: a privilege and our responsibility. Obstetrics \& Ginecology. 1994; 83 (1): 1-4. 